\title{
Coagulation Profile in Perinatal Asphyxia
}

\author{
Murthy $\mathbf{S}^{1}$, Krishnamurthy $\mathbf{V}^{2}$ \\ ${ }^{1}$ Dr Srinivasa Murthy Doreswamy, MBBS, MD (Ped), MRCPCh (UK), Fellowship in Neonatology (Canada) Associate \\ professor in Pediatrics, ${ }^{2}$ Dr Vani Krishnamurthy, MBBS, MD (Path), Assistant professor in Pathology. Both are affiliated \\ to JSS Medical College, JSS University, Mysuru, Karnataka, India.
}

Address for Correspondence: Email: Dr Srinivasa Murthy Doreswamy, No 70, PRAKRUTHI, BEML $2^{\text {nd }}$ stage, Rajarajeshwari Nagara, Mysuru, Karnataka, India, Email: drdsrinivasa@ gmail.com

\begin{abstract}
Introduction: Perinatal asphyxia is a common problem with devastating consequences in neonatal practice. Bleeding due to coagulation abnormality is very common and is potentially treatable. Various mechanisms including liver dysfunction, unresponsiveness to Vitamin $\mathrm{K}$ and consumption of coagulation factors all have been implicated in severe bleeding in asphyxiated neonates. The magnitude of this problem and therapeutic window if present has not been well studied. Methods: this was a prospective study undertaken in a tertiary care centre. 20 babies were recruited for the study. Asphyxia was defined on pragmatic basis of Apgar score and need for resuscitation followed by clinical course. The blood sample was collected in citrated tubes and analysed for coagulation parameters. Results: $55 \%$ of our subjects were having deranged coagulation. APTT was the commonest deranged parameters in all our subjects (11/20). PT was deranged in only 2 of 20 patients. Patients who had deranged coagulation had poor outcomes. Coagulation abnormalities within first 24 hours had a positive predictive value of $18 \%$ and negative predictive value of $100 \%$ with respect to later bleeding. Conclusion: Deranged coagulation is very common in asphyxiated neonates and is associated with poor outcome.
\end{abstract}

Key words: Birth Asphyxia, Neonate, Coagulation

\section{Introduction}

Perinatal asphyxia has been recognized as common cause of newborn death since few centuries. In $19^{\text {th }}$ century, the efforts were aimed towards resuscitation of such babies to bring them back to life. Since then advancement in neonatal care has led to various strategy to improve the intact come of these babies.

An understanding of association of bleeding with asphyxia can open up opportunities for life saving interventions. Recognizing coagulation status earlier to clinical bleeding may have a role in early intervention to prevent life threatening bleeding. There are only few studies on coagulation status of asphyxiated newborns from few decades ago. $[1,2]$.

Birth asphyxia is a condition with variable degree of damage to most of the organs in the body. Coagulation being very delicately balanced system does get

Manuscript received: $1^{\text {st }}$ Oct 2015

Reviewed: $8^{\text {th }}$ Oct 2015

Author Corrected; $17^{\text {th }}$ Oct 2015

Accepted for Publication: $2^{\text {nd }}$ Nov 2015 deranged during birth asphyxia. Studies have shown that there are multiple mechanism including liver dysfunction [3] and consumption of coagulation [4] factors as cause of deranged coagulation in these babies.

Animal models were developed and studied to understand the pathogenesis of such a derangement. Experiment has shown that hypoxia alone without changes in blood pressure, carbon dioxide and $\mathrm{P}^{\mathrm{H}}$ will not result in changes in coagulation system [5]. However in practice all these components are almost always seen in variable proportions and end up in altering the coagulation mechanism.

Vitamin $\mathrm{K}$ is precarious in most of the newborns. This is further accentuated in preterm, ill and asphyxiated babies. Hence all the babies receive Vitamin $\mathrm{K}$ at birth. It is interesting to note that the response to vitamin $\mathrm{K}$ in elevating the coagulation factors is blunted in asphyxiated babies, which could be one other 
mechanism of developing coagulation derangement [6]. In view of this observation, few clinicians recommended giving fresh frozen plasma to all newborn asphyxiated babies with an aim of reducing intracranial bleed and death consequent to it. One controlled study which addressed this issue failed to show any significant impact of such an intervention in prevention of intracranial bleed and mortality [7].

Various studies conducted for different purposes have also shown that coagulation system gets deranged in birth asphyxia and other causes of respiratory failure [8, 9].

Bleeding due to coagulation dysfunction in birth asphyxia is well known. However the magnitude of this problem has not been well studied. Few of the studies which tried to address this problem have included very few infants to draw any meaningful conclusions. There have been significant advancements in managing asphyxiated neonate over last few decades. This brings us the opportunity to relook at this important problem and formulate management strategy.

Primary objective: To estimate the magnitude of coagulation derangement in babies who suffered birth asphyxia

\section{Material and Methods}

Study design and setup: This was a prospective observational study conducted in the department of Pediatrics and department of Pathology, JSS hospital Mysore, Karnataka, India. This is a tertiary care center.

Sample size: This was a pilot study. We had an average of 5 admissions per month to our unit who would fit into the inclusion criteria. Hence we considered a convenient sample size of 20 cases which could be collected over a period of 6 to 8 months.

\section{Inclusion criteria:}

Babies of all gestational age who had birth asphyxia were included.

Birth asphyxia was diagnosed on multiple pragmatic criteria.

The diagnosis of birth asphyxia was considered if

a. APGAR score $<7$ at 5 mins

b. APGAR score of $<5$ at one min and later developed Hypoxic ischemic encephalopathy or renal failure or Necrotizing enterocolitis or Respiratory distress needing support. In all these cases sepsis was excluded by blood culture c. There was history of fetal distress and baby needed resuscitation at birth with bag and mask. Later arterial blood gas done within an hour revealed metabolic acidosis/Hypoxia/Hypercarbia or in combination of these. Or if the infants developed features of asphyxia as mentioned in criteria ' $b$ '.

This criterion was considered as many babies were referred from peripheral hospitals with no documentation of APGAR scores or APGAR scores were not reliable.

\section{Exclusion criteria:}

1. Severe congenital anomalies

2. Babies developing bleeding before 6 hours of life (this criterion was selected with an assumption that coagulation derangement occurs as cascade of multiple mechanisms following an acute asphyxial event which takes few hours to complete.)

Consent: At admission all the parents were informed of the diagnosis and plan of management. They were updated about various tests including blood tests needed for management. We informed that this sample would be taken along with other blood tests needed for clinical management of the baby. Consent was obtained in the official admission document.

Data collection: On inclusion, we have followed standard procedures in managing birth asphyxia and no new therapeutic intervention has been done at this stage. We have obtained blood for coagulation studies between 6 to 24 hours after birth along with other blood sample needed for management of the baby.

Sample collection and processing: Sample was collected in the special tube supplied by the department of pathology. This was a transparent conical tube with 100 microliters of $3.8 \%$ freshly prepared sodium citrate solution filled in the tube. The venous blood was dripped into the tube to make up to the volume of $1 \mathrm{ml}$ marked on the tube. This was secured with a tight cap, labeled and immediately. The samples, thus sent were processed within an hour of the receipt.

Interpretation of results: PT and INR were reported by the lab. INR of > 1.5 was taken as prolonged for further management. APTT reported by the lab was compared to control and considered prolonged if it was greater than control.

Statistical analysis: Continuous variables are expressed as mean and standard deviation. Categorical variables were expressed as proportions. Predictive values were computed for coagulation derangement. 


\section{Results}

Total of 20 babies were recruited into the study. 13 of them were females and 7 were males. 18 babies were born at term and two were preterm. One of the preterm was of 34 weeks gestation and other was 32 weeks of gestation. Median APGAR at 1 minute was 4 with interquartile range between 3 and 5, at five minutes was 7 with interquartile range between 7 and 8 of these babies developed hypoxic ischemic encephalopathy and out of them 3 had renal dysfunction as well. 3 babies had GI bleed during the time of sampling and 2 of them turned out to be having prolonged coagulation. Mean (SD) prothrombin time was 16.5 (6.2) seconds, Mean (SD) INR was 1.25(0.37) and Mean (SD) APTT was 46 (18.7) seconds. Prolonged APTT compared to control was seen in 11 of 20 babies.

There were 2 babies who had high INR (>1.5). Both of them also had prolonged APTT. The details of coagulation profile and related outcome is depicted in Table 1.

\section{Table 1: Coagulation profile and related outcome}

\begin{tabular}{|l|c|}
\hline & $\mathrm{n}$ \\
\hline Prolonged INR & 2 \\
\hline Prolonged APTT & 11 \\
\hline PT alone deranged & 0 \\
\hline APTT alone deranged & 9 \\
\hline Deranged coagulation associated bleeding & 2 \\
\hline Deranged coagulation and later bleeding & 2 \\
\hline Normal coagulation and later bleeding & 1 \\
\hline Deranged coagulation and poor outcome & 3 \\
\hline Normal coagulation and poor outcome & 0 \\
\hline
\end{tabular}

Figure 1: Coagulation status and the related outcome in neonates with perinatal asphyxia

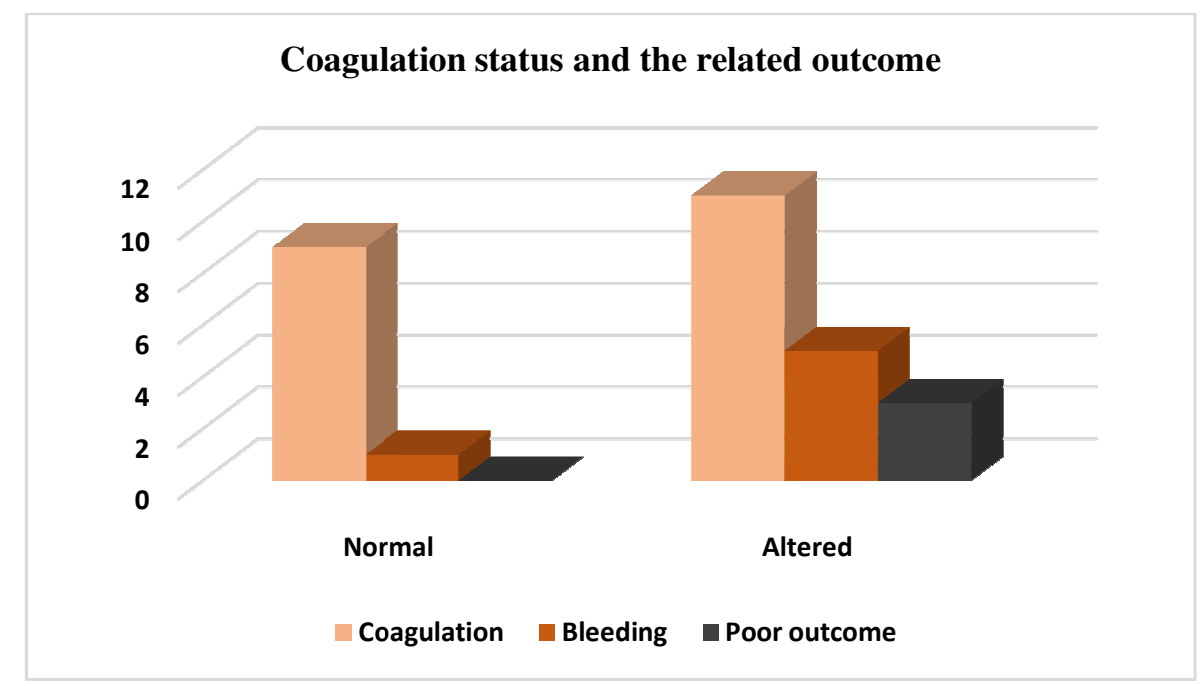

Out of 20 babies 3 had poor outcome. One baby died due to intracranial bleed. Rest 2 of them was very sick when their parents decided to withdraw support and left against medical advice. All 3 of the babies with poor outcome had abnormal coagulation in spite of appropriate treatment. Clinical bleeding with its disastrous consequence being the point of concern, the positive predictive value of abnormal coagulation (before clinical bleeding) is $18 \%$ and negative predictive value is $100 \%$.

\section{Discussion}

In this study we have noted that there is significant proportion of babies develop abnormal coagulation status after first few hours of life. Birth asphyxia is an acute event causing enormous hemodynamic rearrangement in the body. The significant change in the hemodynamics is the diving reflex where in the 
circulation is maintained to vital organs and less vital organs suffer ischemia. This results in liver getting under perfused which may result in impaired function. As the consequence this, the coagulation system gets variably affected. Our study has reinforced this fact by noting higher proportion of babies with severe asphyxia has coagulation abnormality. 3 babies had gastrointestinal bleed which could be due to factors like stress along with altered coagulation.We have noted that more than half of the babies developed altered coagulation. Similar trend have been noted in earlier studies [1,9]. Study by Chadd MA, et al, have revealed all the babies who have undergone asphyxia had mean values of the coagulation parameters significantly lower to controls. They have also noted that the degree of derangement is proportional to severity of asphyxia.

Hambleton and Appleyard [7] did a controlled study to determine the effect of Fresh frozen plasma on coagulation status of asphyxiated infants. They have shown the only parameter that improved was partial thromboplastin time. They did not note any significant improvement in outcome with intracranial bleed as the target parameter. $30 \%$ of their treated patients died which was similar to untreated group. In our study the predominant alteration was APTT. Two of the babies who had high INR also had increased APTT. Only $18 \%$ had poor outcome. This lower incidence of poor outcome in our study probably reflects the advance in overall neonatal management strategies.Low positive predictive value of $18 \%$ and high negative predictive value of $100 \%$ is quite encouraging to consider routine assessment of coagulation status in asphyxiated babies.

\section{Conclusion}

Deranged coagulation status is very common in asphyxiated newborn babies which may precede clinical bleeding. Appropriate action at this stage may be helpful

\section{Limitation:}

1. Sample size is small

2. Severity of asphyxial insult is not compared with magnitude of coagulation derangement.
Funding: None

Conflict of Interest: None

Permission from IRB: Yes

\section{References}

1. Chessells JM, Wigglesworth JS. Coagulation studies in severe birth asphyxia. Arch Dis Child. 1971 Jun;46(247):253-6.

2. Dorothy RB, Michael AS, William EH. Coagulation studies in extremely premature infants. Pediatric research 1979;13:1330-35.

3. Godambe SV, Udani RH, Malik S, Kandalkar BM. Hepatic profile in asphyxia neonatorum. Indian Pediatr. 1997 Oct;34(10):927-30.

4. Chadd MA, Elwood PC, Gray OP, Muxworthy SM. Coagulation defects in hypoxic full-term newborn infants. Br Med J. 1971 Nov 27;4(5786):516-8.

5. Kisker CT, Robillard JE, Clarke WR. Blood coagulation changes after hypoxemia: a fetal lamb model. Pediatr Res. 1982 Jan;16(1):8-12.

6. Appleyard WJ, Cottom DG. Effect of asphyxia on thrombotest values in low birthweight infants. Arch Dis Child. 1970 Oct;45(243):705-7.

7. Hambleton G, Appleyard WJ. Controlled trial of fresh frozen plasma in asphyxiated low birthweight infants. Arch Dis Child. 1973 Jan;48(1):31-5.

8. Watkins MN, Swan S, Caprini JA, Gardner TH, Zuckerman L, Vagher JP. Coagulation changes in the newborn with respiratory failure. Thromb Res. 1980 Jan 1-15;17(1-2):153-75.

9. Ibrahim ZK. Maduhu, Karim P.Manji, Roger L.Mbise. Perinatal risk factors for neonatal bleeding at the Muhimbili national hospital Dar - Es - Sakaam, Tanzania.Tanzania medical journal 2004 Jun;19(2):1-3.

\section{How to cite this article?}

Murthy S, Krishnamurthy V. Coagulation Profile in Perinatal Asphyxia. Pediatr Rev: Int J Pediatr Res 2015;2(4):3740.doi:10.17511/ijpr.2015.i04.01 